\title{
Root proximity of the anchoring miniscrews of orthodontic miniplates in the mandibular incisal area: Cone-beam computed tomographic analysis
}

\author{
Do-Min Jeong ${ }^{\mathrm{a}}$ (1) \\ Song Hee $\mathrm{Oh}^{\mathrm{a}}$ (1) \\ HyeRan $\mathrm{Choo}^{\mathrm{b}}$ \\ Yong-Suk Choi ${ }^{\mathrm{a}}$ \\ Seong-Hun Kim ${ }^{\mathrm{c}}$ \\ Jin-Suk Lee ${ }^{\mathrm{d}}$ \\ Eui-Hwan Hwang ${ }^{\mathrm{a}}$ (1)
}

${ }^{a}$ Department of Oral and Maxillofacial Radiology, Graduate School, Kyung Hee University, Seoul, Korea

${ }^{b}$ Department of Surgery, Division of Plastic and Reconstructive Surgery, Craniofacial and Airway Orthodontic Clinic, Stanford University School of Medicine, Lucile Packard Children's Hospital, Palo Alto, CA, USA

'Department of Orthodontics, Graduate School, Kyung Hee University, Seoul, Korea

${ }^{\mathrm{d}}$ Department of Dental Education, Graduate School, Kyung Hee University, Seoul, Korea

\begin{abstract}
Objective: This outcome analysis study evaluated the actual positions of the orthodontic miniplate and miniplate anchoring screws (MPASs) and the risk factors affecting adjacent anatomic structures after miniplate placement in the mandibular incisal area. Methods: Cone-beam computed tomographic images of 97 orthodontic miniplates and their 194 MPASs (diameter, $1.5 \mathrm{~mm}$; length, $4 \mathrm{~mm}$ ) in patients whose miniplates provided sufficient clinical stability for orthodontic treatment were retrospectively reviewed. For evaluating the actual positions of the miniplates and analyzing the risk factors, including the effects on adjacent roots, MPAS placement height (PH), placement depth (PD), plate angle (PA), mental fossa angle (MA), and root proximity were assessed using the paired $t$-test, analysis of variance, and generalized linear model and regression analyses. Results: The mean PDs of MPASs at positions 1 (P1) and 2 (P2) were $2.01 \mathrm{~mm}$ and $2.23 \mathrm{~mm}$, respectively. PA was significantly higher in the Class 111 malocclusion group than in the other groups. $\mathrm{PH}$ was positively correlated with MA and PD at P1. Of the 97 MPASs at P1, 49 were in the no-root area and 48 in the dentulous area; moreover, 19 showed a degree of root contact (19.6\%) without root perforation. All MPASs at P2 were in the no-root area. Conclusions: Positioning the miniplate head approximately $1 \mathrm{~mm}$ lower than the mucogingival junction is highly likely to provide sufficient $\mathrm{PH}$ for the $\mathrm{P} 1-$ MPASs to be placed in the no-root area.
\end{abstract}

[Korean J Orthod 2021;51(4):231-240]

Key words: Cone-beam computed tomography, Miniplate, Anchorage, Orthodontic treatment

Received November 10, 2020; Revised March 2, 2021; Accepted March 12, 2021.

Corresponding author: Eui-Hwan Hwang.

Professor, Department of Oral and Maxillofacial Radiology, Graduate School, Kyung Hee University, 26 Kyungheedae-ro, Dongdaemun-gu, Seoul 02447, Korea.

Tel +82-2-958-9405 e-mail hehan@khu.ac.kr

Do-Min Jeong and Song Hee Oh contributed equally to this work as co-first authors.

How to cite this article: Jeong DM, Oh SH, Choo HR, Choi YS, Kim SH, Lee JS, Hwang EH. Root proximity of the anchoring miniscrews of orthodontic miniplates in the mandibular incisal area: Cone-beam computed tomographic analysis. Korean J Orthod 2021;51:231-240.

(C) 2021 The Korean Association of Orthodontists.

This is an Open Access article distributed under the terms of the Creative Commons Attribution Non-Commercial License (http://creativecommons.org/licenses/by-nc/4.0) which permits unrestricted non-commercial use, distribution, and reproduction in any medium, provided the original work is properly cited. 


\section{INTRODUCTION}

The use of orthodontic miniscrews to achieve absolute anchorage has now become a staple part of orthodontic treatment strategies. ${ }^{1-3}$ Orthodontic miniscrews are inexpensive and easy to place and remove. Moreover, their small size allows orthodontists to place them at various locations inside the oral cavity for different applications. However, an orthodontic miniscrew placed in an interradicular space can interfere with anteroposterior tooth movement in some cases. In addition, the initial stability of a miniscrew that contacts the root of a tooth can be jeopardized. ${ }^{4,5}$ Miniscrews can induce molar intrusion and reduce the side effects that occur with dental anchorage. However, simple miniscrew anchorage is vulnerable to failure and perhaps incapable of bearing the heavier forces or moments that might be necessary for molar control during uprighting. ${ }^{6}$ In contrast, orthodontic miniplates (OMPs) can serve as possible alternatives to orthodontic miniscrews in some anatomically complex areas. Miniplates are often anchored using miniplate anchoring screws (MPASs) and can be placed at locations much lower than those reachable using orthodontic miniscrews, sometimes completely avoiding interradicular spaces. ${ }^{6-9} \mathrm{ln}$ addition, an OMP anchored by at least two MPASs provides an increased contact surface between the plate and the bone. ${ }^{5}$ When compared to orthodontic miniscrews, OMPs that are appropriately installed can endure more demanding orthodontic or orthopedic forces for a longer time. ${ }^{10}$ However, conventional OMPs have shortcomings such as the need for flap surgery, postoperative medication, and post-placement complications despite stable treatment results. ${ }^{8,9}$

OMPs with round tubes are intended to function independently of regular fixed orthodontic appliances on the teeth as well as indirect anchorage sources (Figure
1). ${ }^{8-11}$ Such OMPs are flexible and have a 0.036-inch tube-shaped head that remains exposed to the oral cavity during active orthodontic treatment. Two to three MPASs are often required to stabilize an OMP.,12 The maxillary posterior region is one of the most common locations used for the placement of OMPs. Kim et al. ${ }^{12}$ evaluated the position and associated risk factors in the use of OMPs and their 4-mm MPASs in the posterior maxilla by employing three-dimensional (3D) cone-beam computed tomography (CBCT). The average bone penetration depth of an MPAS was $2.48 \mathrm{~mm}$, and it did not cause any clinically significant damage to the adjacent roots. The mandibular incisal area is another commonly used location for miniplate placement, since this area is favorable for many direct and indirect orthodontic biomechanics. ${ }^{10-12} \ln$ addition, this area is favored because the surgical placement procedure is relatively simple because of easy access and minimal anatomic limitations. Despite the frequent placement and application of OMPs in the mandibular incisal area, very limited data have been reported on what contributes to their successful clinical stability in this area.

The purpose of this outcome analysis study was to use retrospectively collected CBCT data to evaluate the actual positions of OMPs and their MPASs placed in the mandibular incisal area, as well as to assess the risk factors and other effects of the 4-mm MPASs on adjacent anatomic structures after placement in patients who had unfavorable anatomic features hindering standard single miniscrew anchorage.

\section{MATERIALS AND METHODS}

\section{Patients}

Orthodontic patient records from 2009 to 2019 at the Kyung Hee University Dental Hospital were reviewed to
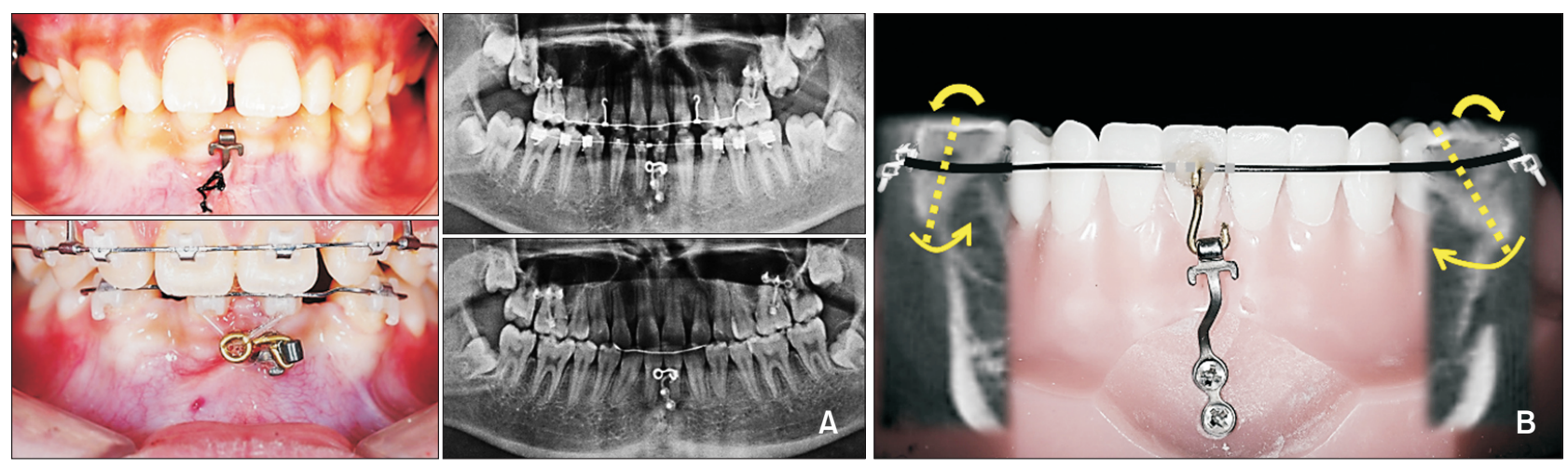

Figure 1. Clinical application of the orthodontic miniplate (OMP) used in this study. A, OMP for lower intrusion and molar protraction. Intraoral photographs and panoramic radiographs show multidirectional tooth movement achieved using a bendable OMP. B, Schematic illustration of an OMP combined with a reverse curve NiTi application for lower posterior uprighting with intrusion. 
identify patients whose treatment utilized a 1-shaped OMP in the mandibular incisal area during orthodontic treatment. An 1-shaped OMP with two MPAS holes was the miniplate of choice for the patients in the study (C-tube plate, JBOP-001-1; Jin Biomed Co., Bucheon, Korea). The study included patients with successful miniplate installation in the mandibular incisal area. Installation success was defined as the provision of sufficient anchorage by the miniplate during orthodontic treatment lasting more than 6 months without any complications. The exclusion criteria were as follows: (1) insufficient quality radiographic images because of patient movement, metal artifact, or head position; (2) miniplate failure because of detachment, loosening, abscess, tissue irritation, or wrong insertion direction; and (3) complications such as infection, transient tissue irritation, loosened but functional MPASs, MPAS fracture, or localized inflammation. ${ }^{9}$ In total, 97 patients, including 82 requiring lower anterior intrusion or vertical dimension control, three requiring anchorage for molar protraction, and 12 requiring lower posterior uprighting, were selected for the study. They included 38 male and 59 female patients. Their mean age was $22.16 \pm 7.84$ years, with the ages ranging from 11 to 50 years old. Thirty patients (mean age, $23.3 \pm 8.27$ years) had skeletal Class 1 malocclusion, 50 (mean age, $21.9 \pm$ 8.26 years) had Class 11 malocclusion, and 14 (mean age, $20.5 \pm 4.65$ years) had Class 111 malocclusion at the time of miniplate installation. This study was approved by the Institutional Review Board of Kyung Hee University (IRB-KH-DT-19038).

\section{OMP placement protocol}

One OMP was placed per patient on the labial aspect of the anterior mandibular bone between the two central incisors (Figure 2). The MPAS hole close to the OMP head was designated as position $1(\mathrm{P} 1)$ and the hole further away from the head as position 2 (P2) (Figure 2A). Each MPAS was $4 \mathrm{~mm}$ in length and $1.5 \mathrm{~mm}$ in diameter. The OMP placement protocol has been previously described in detail." Briefly, a flat l-shaped OMP was bent using a sharp-tipped Weingart plier (Osung MND, Gimpo, Korea) according to the shape of the patient's mandibular symphysis as seen on the cephalometric image. After administering local anesthesia, an approximately 7-mm vertical incision was made at the vestibule between the two mandibular incisors by using a \# 15 blade. The periosteum was carefully lifted using a periosteal elevator and the OMP was placed on the bone with a self-drilling MPAS at P2, while the miniplate head was positioned at the level of the mucogingival junction (Figure 2B and 2C). The second MPAS was then placed at P1, avoiding the teeth (Figure 2D). The incision was sutured with 4-0 silk (Figure 2E). The miniplate was loaded with orthodontic level forces after 1 week and was retained successfully for 6 months or longer in all patients.

\section{CBCT protocol and measurement}

CBCT scans were acquired with a voxel size of 0.39 $\mathrm{mm}^{3}$ (Alphad Vega; Asahi Roentgen, Kyoto, Japan) and formatted using a Picture Archiving and Communication System (Infinite, Seoul, Korea). The obtained data were imported as digital imaging and communications in medicine (DICOM) files using OnDemand 3D ${ }^{\mathrm{TM}}(\mathrm{Cy}-$
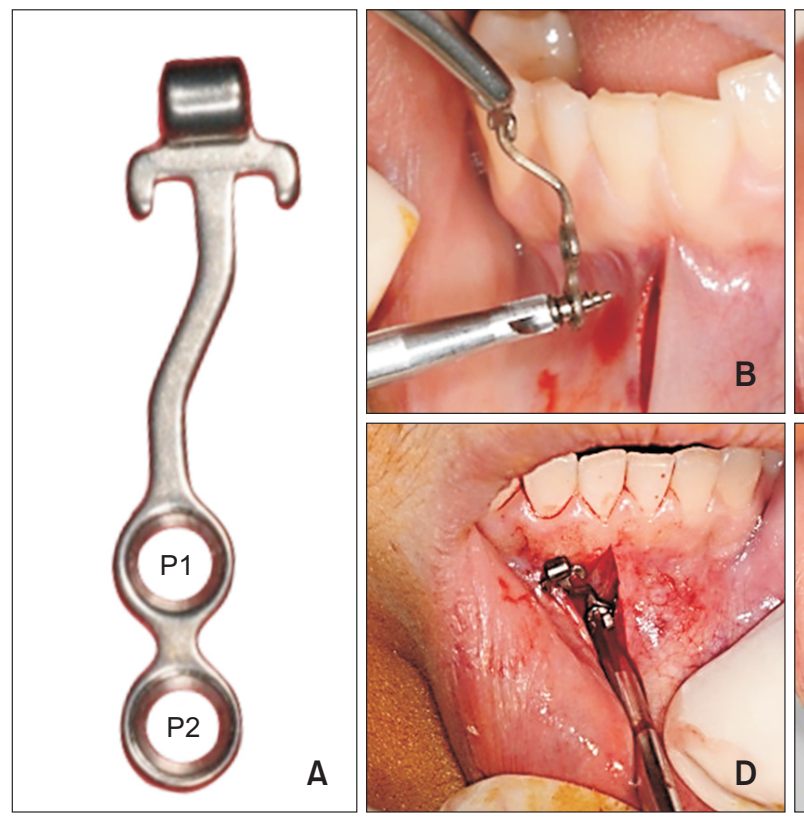

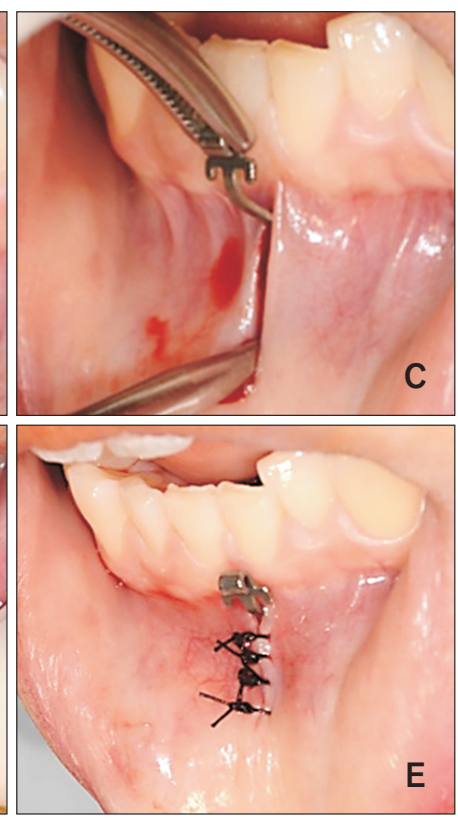

Figure 2. Placement protocol of the orthodontic miniplate (OMP). A, The I-type OMP with two holes and a tube-shaped head. B, The OMP is placed with self-drilling miniplate anchoring screws (MPASs). C, The OMP is placed in the incised area. D, MPASs are fixed to the cortical bone under the mucosa by using a manual screwdriver. $\mathbf{E}$, The incised area is sutured with 4-0 silk. $\mathrm{P} 1$, position $1 ; \mathrm{P} 2$, position 2. 
bermed, Daejeon, Korea). Two examiners performed all of the measurements on the CВCT images by using the same computer and screen (resolution, 1,920 × 1,440 pixels) under ambient room lighting conditions. The sagittal plane was drawn perpendicular to the occlusal plane, which was established by the occlusal surfaces of the mandibular first molars. Miniplate placement height (PH), placement depth (PD), plate angle (PA), mental fossa angle (MA), and root proximity (RP) were assessed. $\mathrm{PH}$ was measured as the distance from the cementoenamel junction (CEJ) to the center of P1 on the adjusted sagittal image (Figure $3 \mathrm{~A}$ ). PD was measured as the bone thickness an MPAS penetrated on the sagittal plane (Figure 3B); PD1 was the thickness at P1 and PD2 at P2. PA was defined as the angle of the step-up bend on the miniplate that was made by an orthodontist based on the soft- and hard-tissue anatomic features at the incision site (Figure 3C). MA was defined as an angle made by the two tangents on the sagittal plane, one from the most prominent midpoint of the chin and the other from the most prominent point on the labial root surface (Figure 3D). When an MPAS contacted the adjacent root surface or impinged the periodontal ligament (PDL) of the mandibular incisors in any of the three planes (axial, cross-sectional, or sagittal), it was defined as $\mathrm{RP}^{13}$ (Figure 4). MPASs were divided into four groups according to RP: group 1 with frontal overlap, group 2 with an interdental space, group 3 with lateral contact, and group 4 in a no-root area. Group 1 was further subdivided into no contact (1a), frontal contact (1b, MPAS contacted the labial surface of the mandibular incisor), and root perforation (1c) (Figure 4B).

\section{Statistical analysis}

To determine interexaminer reproducibility, the same examiners repeated the measurements on 20 sets of randomly selected CBCT data after a 2-week interval. The resultant intraclass correlation coefficient (ICC, 0.75$0.88 ; p<0.001$; Kappa value, 0.9$)$ and interobserver coefficient (ICC, 0.84-0.97; $p<0.001$; Kappa value, 0.85) indicated high reliability. A paired $t$-test was used to evaluate the differences between PD1 and PD2, which
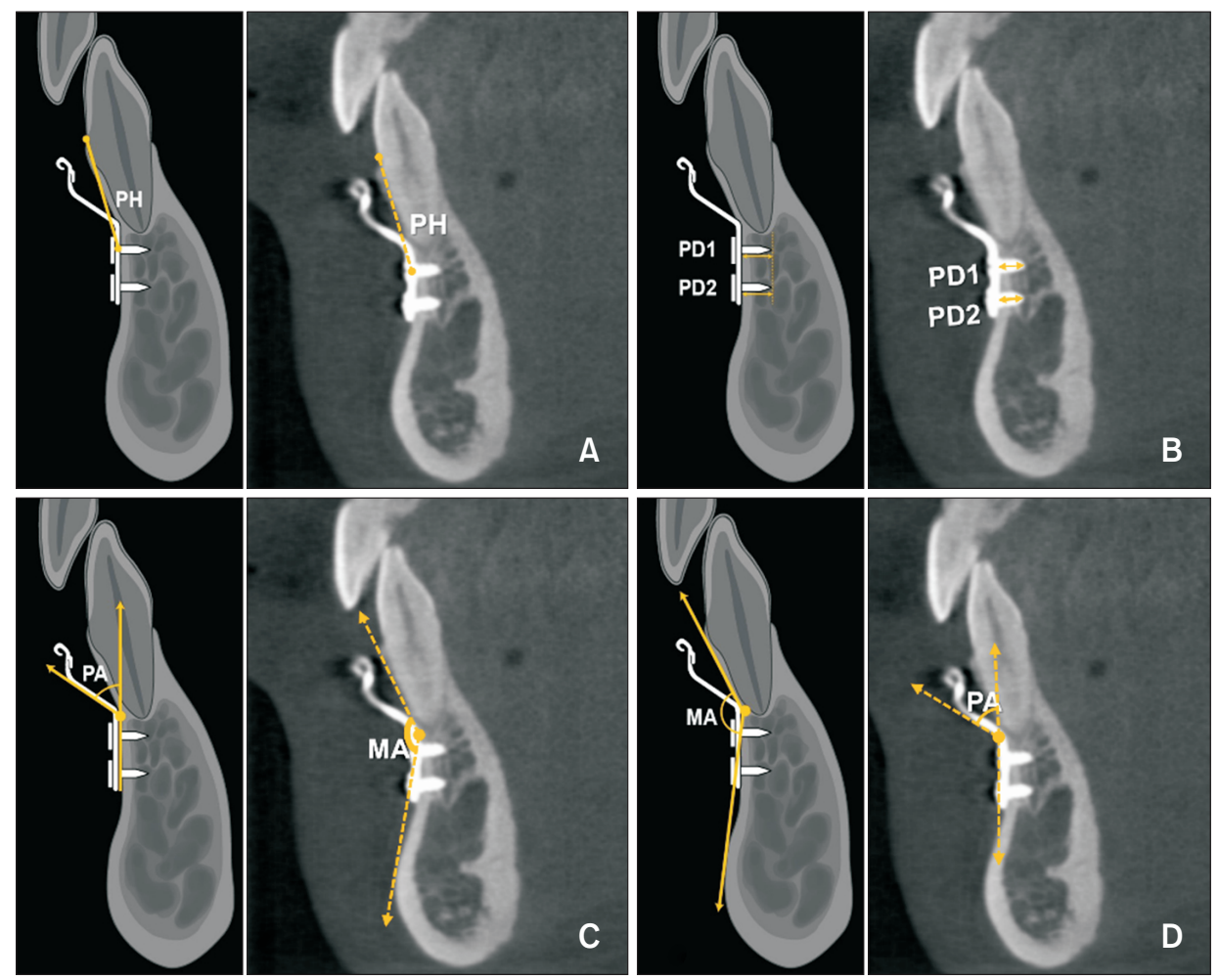

Figure 3. Schematic illustration and actual cone-beam computed tomographic image of the orthodontic miniplate (OMP) in the anterior mandible. A, The vertical distance from the cementoenamel junction to the center of the miniplate anchoring screw (MPAS) at position 1 (P1) (placement height, PH). B, Distance from the outer surface of the labial cortical bone to the tip of the MPASs at P1 (PD1) and P2 (PD2). C, The angle of the step-up bend on the OMP that was made by an orthodontist based on the soft- and hard-tissue anatomic features of the incision site (plate angle, PA). D, The angle made by the two tangents on the sagittal plane, with one from the most prominent midpoint of the chin and the other from the most prominent point on the labial root surface (mental fossa angle, MA). 
A

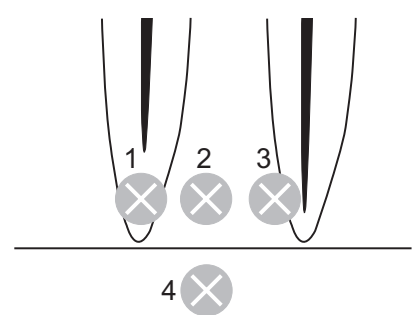

B

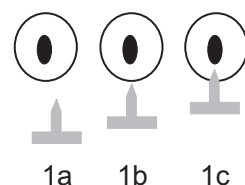

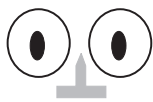

2

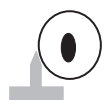

3

Figure 4. Schematic illustration showing the classification of root proximity according to the location of the miniplate anchoring screw (MPAS) at position 1. A, Frontal view: group 1, frontal overlap (no contact [1a], frontal contact [1b, the MPAS contacts the labial surface of the mandibular incisor], and root perforation [1c]); group 2, interdental space; group 3, lateral contact; and group 4, no-root area. B, Axial view: 1a, no contact; 1b, frontal contact; 1c, root perforation; 2 , interdental space; 3, lateral contact.

Table 1. Various miniplate measurements

\begin{tabular}{lc}
\hline Variable & Value \\
\hline PH $(\mathrm{mm})$ & $11.42 \pm 2.05$ \\
PD1 $(\mathrm{mm})$ & $2.01 \pm 0.43^{\mathrm{a}}$ \\
PD2 $(\mathrm{mm})$ & $2.23 \pm 0.38^{\mathrm{b}}$ \\
PA $\left(^{\circ}\right)$ & $54.16 \pm 11.55$ \\
$\mathrm{MA}\left(^{\circ}\right)$ & $140.77 \pm 8.41$ \\
\hline
\end{tabular}

Values are presented as mean \pm standard deviation. Paired $t$-test was performed.

$\mathrm{PH}$, vertical distance from the cementoenamel junction to the center of the miniplate anchoring screw (MPAS) at position 1; PD, distance from the outer surface of the labial cortical bone to the tip of the MPASs at positions 1 (PD1) and 2 (PD2); PA, angle of the miniplate placed on the mandibular symphysis; MA, angle made by the two tangents, with one from the most prominent midpoint of the chin and the other from the prominent point on the buccal root surface.

${ }^{\mathrm{a}, \mathrm{b}}$ Statistically significant differences between PD1 and PD2; $p<0.05$.

followed a normal distribution at the 5\% significance level. Age and sex differences were disregarded. Generalized linear model and regression analyses were used to evaluate the associations of PH, PD, PA, and MA. Analysis of variance (ANOVA) and multivariable comparison using Bonferroni post-hoc analysis were used to compare differences between $\mathrm{PH}, \mathrm{PD}, \mathrm{PA}$, and MA according to the skeletal malocclusion classification or RP. All statistical analyses were performed using SAS software ver. 8.0 (SAS Institute, Cary, NC, USA).

\section{RESULTS}

Tables 1 and 2 show the means and standard deviations of $\mathrm{PH}, \mathrm{PD}, \mathrm{PA}$, and MA of the current outcome analysis. PD1 values $(2.01 \pm 0.43 \mathrm{~mm})$ were significantly lower than PD2 values $(2.23 \pm 0.38 \mathrm{~mm} ; p<0.05)$. ANOVA revealed that PA values in the Class 111 group
$(63.32 \pm 8.55)$ were significantly lower than those in the Class $1(50.57 \pm 9.25)$ and Class 11 groups (54.24 \pm 12.59). No differences were observed in $\mathrm{PH}, \mathrm{PD}$, and $\mathrm{MA}$ among the different malocclusion groups.

Spearman's correlation analysis to examine the mutual effects among the variables showed that $\mathrm{PH}$ had a positive correlation with MA and a negative correlation with PA, while PD1 values had a negative correlation with PA and a positive correlation with $\mathrm{PH}$. The coefficient (estimate) of multivariate regression analysis adjusted by sex and age was confirmed to be consistent with the trend indicated by Spearman's correlation coefficient (Table 3).

Table 4 shows the average value of each measured variable and its range according to the RP classification. When assessed using RP, 49 MPASs at P1 were in the no-root area (group 4; Figure 5). Among the 48 MPASs at P1 that were in the dentulous area, 11 showed no contact (group 1a; Figure 5A). Among the MPASs, 19 showed root contact: 10 showed frontal contact (group 1b; Figure 5B) and nine showed lateral contact (group 3; Figure 5C). Moreover, 18 MPASs were in the interdental spaces (group 2; Figure 5D). However, root perforation was absent. No statistically significant differences were observed among PH, PD, PA, and MA according to RP. $\mathrm{PH}$ showed a statistically significant difference between the dentulous and no-root areas (12.73 mm in group 4; $p<0.0001)$.

\section{DISCUSSION}

In this study, we used MPASs with a length of $4 \mathrm{~mm}$ and a diameter of $1.5 \mathrm{~mm}$. Nevertheless, 19 of the 97 MPASs still showed some degree of root involvement, with nine contacting the lateral surface of the root and 10 contacting the labial surface of the root. All root contacts occurred at $\mathrm{P} 1$. In a clinical situation, when an MPAS contacts the root surface, most patients can feel it immediately and report discomfort even under local anesthesia, thereby prompting an immediate alteration 
Table 2. Comparison of miniplate measurements according to skeletal malocclusion classification

\begin{tabular}{lcccc}
\hline \multirow{2}{*}{ Variable } & \multicolumn{4}{c}{ Skeletal classification } \\
\cline { 2 - 5 } & Class I (n= 33) & Class II (n= 50) & Class III (n= 14) & p-value \\
\hline PH (mm) & $11.32 \pm 2.05$ & $11.41 \pm 2.15$ & $11.69 \pm 1.80$ & 0.8841 \\
PD1 (mm) & $1.97 \pm 0.33$ & $2.04 \pm 0.48$ & $2.02 \pm 0.46$ & 0.7208 \\
PD2 (mm) & $2.22 \pm 0.32$ & $2.28 \pm 0.42$ & $2.09 \pm 0.36$ & 0.2567 \\
PA $\left(^{\circ}\right)$ & $50.57 \pm 9.25^{\mathrm{b}}$ & $54.24 \pm 12.59^{\mathrm{b}}$ & $63.32 \pm 8.55^{\mathrm{a}}$ & 0.0039 \\
MA $\left(^{\circ}\right)$ & $142.08 \pm 7.12$ & $139.25 \pm 9.40$ & $143.15 \pm 6.74$ & 0.4629 \\
\hline
\end{tabular}

Values are presented as mean \pm standard deviation.

Kruskal Wallis test with Bonferroni post-hoc analysis was performed.

$\mathrm{PH}$, vertical distance from the cementoenamel junction to the center of the miniplate anchoring screw (MPAS) at position 1; $\mathrm{PD}$, distance from the outer surface of the labial cortical bone to the tip of the MPASs at positions 1 (PD1) and 2 (PD2); PA, angle of the miniplate placed on the mandibular symphysis; MA, angle made by the two tangents, with one from the most prominent midpoint of the chin and the other from the prominent point on the buccal root surface.

${ }^{\mathrm{a}, \mathrm{b}}$ Statistically significant differences between skeletal malocclusion types in PA; $p<0.05$.

Table 3. The correlation coefficient and multiple generalized linear model estimate between the miniplate measurements adjusted by sex and age

\begin{tabular}{|c|c|c|c|c|c|c|c|}
\hline \multirow{3}{*}{$\begin{array}{c}\begin{array}{c}\text { Independent } \\
\text { variable }\end{array} \\
\text { MA }\end{array}$} & \multirow{3}{*}{$\begin{array}{c}\begin{array}{c}\text { Dependent } \\
\text { variable }\end{array} \\
\mathrm{PH}\end{array}$} & \multicolumn{2}{|c|}{ Spearman's correlation } & \multicolumn{4}{|c|}{ Multiple generalized linear model } \\
\hline & & \multirow{2}{*}{$\begin{array}{c}\begin{array}{c}\text { Correlation } \\
\text { coefficient }\end{array} \\
0.28\end{array}$} & \multirow{2}{*}{$\begin{array}{l}\text { p-value } \\
0.0062^{* *}\end{array}$} & \multirow{2}{*}{$\begin{array}{c}\text { Estimate } \\
0.78\end{array}$} & \multicolumn{2}{|c|}{$95 \%$ CI } & \multirow{2}{*}{$\frac{p \text {-value }}{0.001^{* *}}$} \\
\hline & & & & & 0.31 & 1.25 & \\
\hline MA & PA & 0.09 & 0.3994 & -0.01 & -2.85 & 2.83 & 0.996 \\
\hline MA & PD1 & 0.09 & 0.4045 & 0.05 & -0.06 & 0.16 & 0.346 \\
\hline MA & PD2 & 0.12 & 0.2497 & 0.06 & -0.04 & 0.15 & 0.243 \\
\hline $\mathrm{PA}$ & $\mathrm{PH}$ & -0.24 & $0.0189^{*}$ & -0.43 & -0.78 & -0.09 & $0.015^{*}$ \\
\hline $\mathrm{PA}$ & PD1 & -0.21 & $0.0407^{*}$ & -0.08 & -0.16 & -0.01 & $0.035^{*}$ \\
\hline $\mathrm{PA}$ & PD2 & 0.06 & 0.5314 & 0.03 & -0.04 & 0.10 & 0.354 \\
\hline $\mathrm{PH}$ & PD1 & 0.23 & $0.0222^{*}$ & 0.05 & 0.01 & 0.09 & $0.021^{*}$ \\
\hline $\mathrm{PH}$ & PD2 & 0.08 & 0.4209 & 0.01 & -0.03 & 0.05 & 0.538 \\
\hline
\end{tabular}

$\mathrm{PH}$, vertical distance from the cementoenamel junction to the center of the miniplate anchoring screw (MPAS) at position 1; PD, distance from the outer surface of the labial cortical bone to the tip of the MPASs at positions 1 (PD1) and 2 (PD2); PA, angle of the miniplate placed on the mandibular symphysis; MA, angle made by the two tangents, with one from the most prominent midpoint of the chin and the other from the prominent point on the buccal root surface; CI, confidence interval.

${ }^{*} p<0.05,{ }^{* *} p<0.01$.

of the direction of MPAS placement in situ. This might be a reason why no root perforation was noted in this study. Kuroda et al. ${ }^{13}$ reported that miniscrew contact with the root can be a major risk factor in the failure of orthodontic anchorage. They also suggested that sufficient bone remodeling does not occur where screws are in contact with adjacent teeth. In contrast, Kim et al. ${ }^{14}$ reported that localized RP itself might not be a major causal factor of orthodontic mini-implant failure if primary stability is achieved by other contributing factors, such as a large bone-to-implant contact area, loading time, and treatment protocol, which can compensate for the initial RP. Partial root contact by the MPAS at P1 is compensated for by the MPAS at P2 through the miniplate, which provides primary stability to the MPAS at P1.

When the damage caused by root-screw contact is limited to the PDL, the injury will likely be repaired without further consequence. ${ }^{15}$ When the cementum is damaged and the dentin is exposed because of continuous stimulation, biofilm exposure may occur and multinucleated macrophages will colonize and start resorbing the denuded surfaces. If the affected area is large and deep, the tooth can be truly ankylosed. ${ }^{16}$ Since the average PD1 was $2 \mathrm{~mm}$, RP will be much less than that after subtracting the thickness of the labial bone and PDL, 
Table 4. Comparison of miniplate measurements according to root proximity

\begin{tabular}{lccccc}
\hline \multirow{2}{*}{ Variable } & \multicolumn{4}{c}{ Root proximity } & \multicolumn{4}{c}{$\boldsymbol{p}$-value } \\
\cline { 2 - 5 } & No contact $(\mathbf{n}=\mathbf{1 1})$ & Contact $(\mathbf{n}=\mathbf{1 9})$ & Interdental space $(\mathbf{n}=\mathbf{1 8})$ & No-root area $(\mathbf{n}=\mathbf{4 9})$ & $12.73 \pm 1.36^{\mathrm{a}}$ \\
\hline PH $(\mathrm{mm})$ & $10.88 \pm 1.98^{\mathrm{b}}$ & $9.75 \pm 1.69^{\mathrm{b}}$ & $9.96 \pm 1.66^{\mathrm{b}}$ & $2.09 \pm 0.43$ & 0.0001 \\
PD1 (mm) & $1.81 \pm 0.44$ & $1.95 \pm 0.42$ & $2.01 \pm 0.42$ & $2.20 \pm 0.28$ & 0.8296 \\
PD2 (mm) & $2.29 \pm 0.58$ & $2.26 \pm 0.47$ & $2.25 \pm 0.40$ & $57.18 \pm 12.22$ & 0.0823 \\
PA (mm) & $51.59 \pm 11.40$ & $49.39 \pm 9.54$ & $52.56 \pm 10.01$ & $141.06 \pm 8.54$ & 0.4169 \\
MA (mm) & $138.35 \pm 6.47$ & $142.19 \pm 7.81$ & $139.99 \pm 9.88$ & &
\end{tabular}

Values are presented as mean \pm standard deviation.

Kruskal Wallis test with Bonferroni post-hoc analysis was performed.

$\mathrm{PH}$, vertical distance from the cementoenamel junction to the center of the miniplate anchoring screw (MPAS) at position 1; PD, distance from the outer surface of the labial cortical bone to the tip of the MPASs at positions 1 (PD1) and 2 (PD2); PA, angle of the miniplate placed on the mandibular symphysis; MA, angle made by the two tangents, with one from the most prominent midpoint of the chin and the other from the prominent point on the buccal root surface.

${ }^{a, b}$ Statistically significant difference between root proximity in $\mathrm{PH} ; p<0.05$.
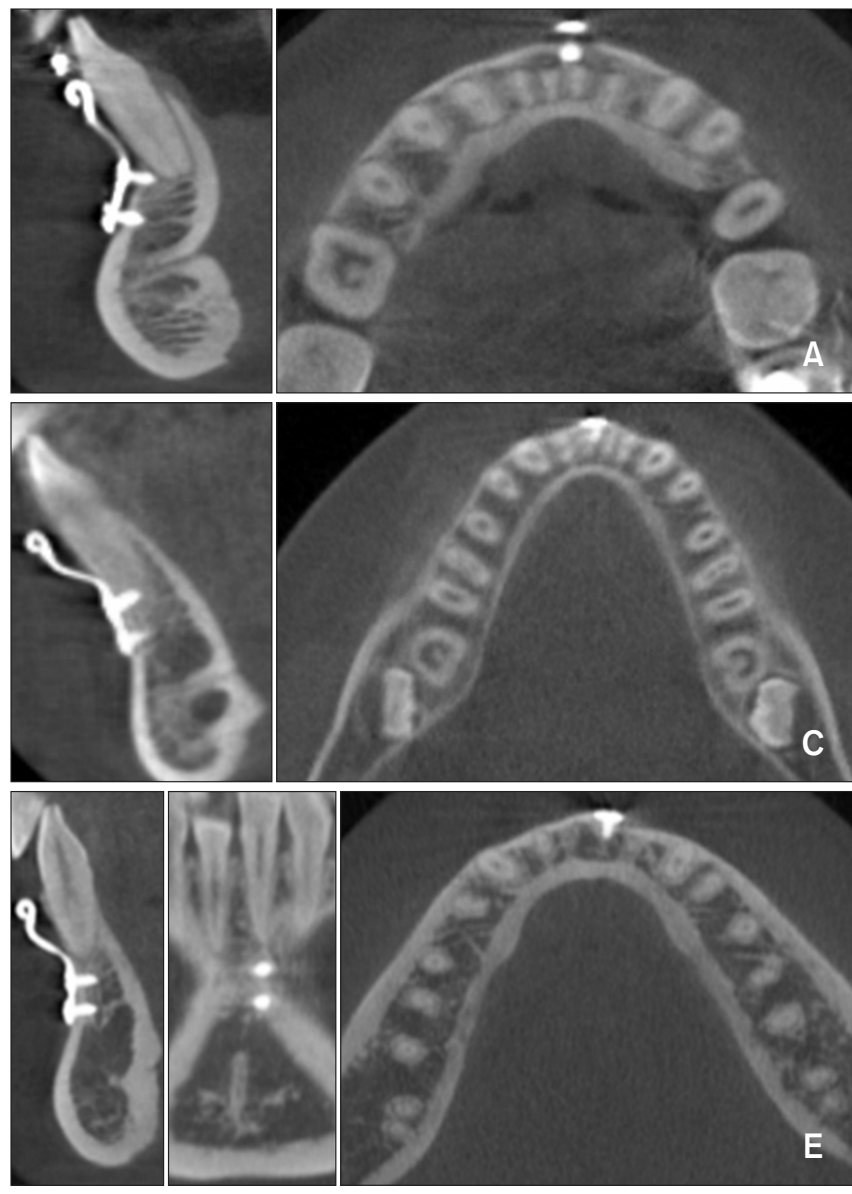

and hence, tooth ankylosis will be unlikely. Therefore, none of the teeth examined in our study showed ankylosis.

Lee et al. ${ }^{17}$ reported that the average safe bone depth to engage an orthodontic miniscrew in the interradicular space of the mandibular incisors was $2.22 \pm 0.6 \mathrm{~mm}$,
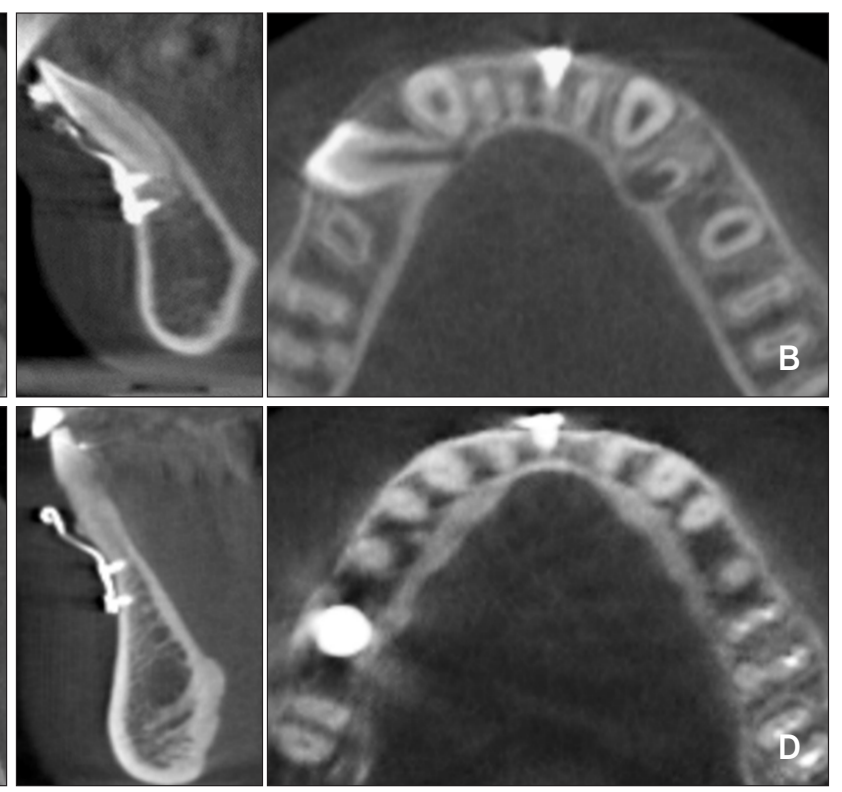

Figure 5. Cone-beam computed tomographic image of the orthodontic miniplate according to the classification of root proximity. A, Group 1 with subdivision a, no contact. B, Group 1 with subdivision b, frontal contact. C, Group 3, lateral contact. D, Group 2, interdental space. E, Group 4, no-root area.

when measured $8 \mathrm{~mm}$ apically from the CEJ. An orthodontic miniscrew with a short PD has less surface area for interdigitation to the bone than does a miniscrew with a long PD and large surface area, and this causes miniscrew failure during the initial stage of biomechanical force application. The current study on successfully 
retained OMPs and their MPASs showed that the average values of PD1 and PD2 were $2.01 \mathrm{~mm}$ and 2.23 $\mathrm{mm}$, respectively, indicating that sufficient bone depth could be obtained in the mandibular anterior area for stabilizing C-tube miniplates. ${ }^{16}$ Statistically significant differences were also observed between PD1 and PD2. All MPASs at P2 were located in the no-root area, thus allowing safe and confident placement of MPASs. Moreover, the tendency of more oblique insertion of MPASs at $\mathrm{P} 2$, which is influenced by the soft-tissue resistance at the entry point of incision, could have contributed to the increased engagement of MPASs into the bone. ${ }^{4}$ Further studies are warranted to elucidate the relationships between the MPAS placement angle and the longterm stability of the miniplate. In our study, the average $\mathrm{PH}$ was $11.42 \pm 2.05 \mathrm{~mm}$ regardless of the malocclusion type. In addition, PH increased as MA increased. PD1 also increased as PH increased. The risk of damaging adjacent roots should decrease when the $\mathrm{PH}$ is large, as it will promote more confident insertion of MPASs at P1. Therefore, it will be reasonable to recommend placing miniplates in such a way that the $\mathrm{PH}$ is increased whenever the anatomic condition of the surgical site allows it. The average $\mathrm{PH}$ in the current study is similar to the average root length of the mandibular central incisor reported by Kim et al., ${ }^{18}$ i.e., $11.56 \mathrm{~mm}$ in male and 10.99 $\mathrm{mm}$ in female patients. Knowing that the average $\mathrm{PH}$ at P2 was $12.73 \mathrm{~mm}$ and that the MPASs were always in the no-root area, the $\mathrm{PH}$ values between $\mathrm{P} 1$ and $\mathrm{P} 2$ can provide a practical reference point for ensuring the placement of MPASs at P1 in no-root areas as much as possible. To ensure safer and more predictable application of OMPs in the mandibular incisal area, the C-tube head can be positioned approximately $1 \mathrm{~mm}$ lower than the mucogingival junction to obtain a sufficient $\mathrm{PH}$ for the MPASs at P1 to be placed in no-root areas. This will also help eliminate the possibility of accidental RP without the need for an unnecessarily large and invasive gingival incision for OMP placement.

MA was included in the study to examine whether it influenced PA depending on the different malocclusion types. MA itself did not show any statistically significant difference among the different malocclusion types. However, PA was significantly higher in patients with Class 111 malocclusion than in those with other malocclusions. Although MA and PA are entirely dependent on individual anatomic variations, one factor that caused the operator to bend the miniplates more in patients with Class 111 malocclusion could be the gingival biotype, as reported by Kaya et al. ${ }^{19}$ They evaluated the width of the keratinized gingiva and gingival biotype in the mandibular anterior region according to the skeletal malocclusion classification. They reported that gingival thickness in the mandibular anterior region was 0.71 $\mathrm{mm}$ with a thin gingival biotype in Class 111 malocclusion, even though the findings did not show statistically significant differences among different malocclusion types. However, our clinical observations indicated that patients with Class 111 malocclusion tended to have greater soft-tissue depths in the mandibular anterior vestibule when an incision was made to place an OMP. Subsequently, this might have prompted the operator to bend the miniplates more to accommodate the soft-tissue thickness in this area. Simultaneously, PA is often determined by the type of necessary biomechanics during orthodontic treatment as well as by anatomic variables. Since a greater $\mathrm{PH}$ assures MPAS placement in no-root areas, the practice of miniplate bending is now evolving toward maintaining the total miniplate length with a minimal step-up bend as close to the OMP head as possible for ensuring favorable gingival emergence of the tube head. The current outcome analysis shows that precontouring the miniplate following the curvature of the mental fossa as viewed on sagittal CBCT images can help maximize the plate-to-bone contact. Gingival emergence bending of the miniplate as close to the OMP head as possible also seems important to ensure patient comfort without compromising $\mathrm{PH}$.

When placing OMPs near dentulous areas, clinical guidelines for MPAS placement can be helpful to ensure the safety and predictability of their use. This is because unlike orthodontic miniscrews, monocortical screws used for fixing the miniplates are intended to not approach or damage the roots while preventing any $3 \mathrm{D}$ tooth movement. $^{20}$

To our knowledge, this is the first study to provide quantifiable data on the actual positions of MPASs that successfully provided clinical stability to OMPs placed in the mandibular incisal area when evaluated three dimensionally. ${ }^{21}$ Nevertheless, this study has some limitations. This was an observational study of clinical outcomes using a single type of miniplate from the same manufacturer and placed in the anterior area. Therefore, factors that have been reported to be associated with RP may be applicable to this specific type of plate. The position of the tube, distance from the screw hole, and plate length might have significant effects on RP. Further large-scale studies including patients whose OMPs did not provide clinically successful stability during orthodontic treatment (in addition to those that were included in the current study) and employing a new grouping strategy in accordance with the vertical craniofacial pattern (because chin morphology varies among hyperdivergent and hypodivergent patients) might be necessary to fully elucidate the risk factors for OMP stability in the mandibular anterior region. 


\section{CONCLUSION}

The findings of this outcome analysis CBCT study showed that the RP of MPASs at P1 seemed to have minimal effects on the successful stabilization of OMPs in the mandibular anterior area. Positioning the tubeshaped head of OMPs approximately $1 \mathrm{~mm}$ lower than the mucogingival junction will likely provide sufficient $\mathrm{PH}$ for the MPASs at P1 to be placed in no-root areas. These findings suggest safe placement of orthodontic miniplates in the mandibular anterior region to ensure predictability outcomes during orthodontic treatment.

\section{CONFLICTS OF INTEREST}

No potential conflict of interest relevant to this article was reported.

\section{ACKNOWLEDGEMENTS}

This article is partly from the PhD thesis of D.M.J.

\section{REFERENCES}

1. Odman J, Lekholm U, Jemt T, Brånemark Pl, Thilander B. Osseointegrated titanium implants--a new approach in orthodontic treatment. Eur J Orthod 1988;10:98-105.

2. Roberts WE, Helm FR, Marshall KJ, Gongloff RK. Rigid endosseous implants for orthodontic and orthopedic anchorage. Angle Orthod 1989;59:247-56.

3. Sadek MM, Sabet NE, Hassan IT. Type of tooth movement during en masse retraction of the maxillary anterior teeth using labial versus lingual biocreative therapy in adults: a randomized clinical trial. Korean J Orthod 2019;49:381-92.

4. Poggio PM, Incorvati C, Velo S, Carano A. "Safe zones": a guide for miniscrew positioning in the maxillary and mandibular arch. Angle Orthod 2006;76:191-7.

5. Chen YJ, Chang HH, Huang CY, Hung HC, Lai EH, Yao CC. A retrospective analysis of the failure rate of three different orthodontic skeletal anchorage systems. Clin Oral Implants Res 2007;18:768-75.

6. Ahn HW, Noh MK, Chung KR, Kim SH, Nelson G. Strategic molar uprighting using the biocreative reverse-curve technique. J Clin Orthod 2020;54:486-94.

7. Sugawara J, Kanzaki R, Takahashi 1, Nagasaka H, Nanda R. Distal movement of maxillary molars in nongrowing patients with the skeletal anchorage system. Am J Orthod Dentofacial Orthop 2006;129: 723-33.

8. Chung KR, Kim YS, Linton JL, Lee YJ. The miniplate with tube for skeletal anchorage. J Clin Orthod
2002;36:407-12.

9. Lee SJ, Lin L, Kim SH, Chung KR, Donatelli RE. Survival analysis of a miniplate and tube device designed to provide skeletal anchorage. Am J Orthod Dentofacial Orthop 2013;144:349-56.

10. Ahn HW, Chung KR, Kang SM, Lin L, Nelson G, Kim $\mathrm{SH}$. Correction of dental Class 111 with posterior open bite by simple biomechanics using an anterior C-tube miniplate. Korean J Orthod 2012;42:270-8.

11. Chung KR, Kim SH, Kang YG, Nelson G. Orthodontic miniplate with tube as an efficient tool for borderline cases. Am J Orthod Dentofacial Orthop 2011;139:551-62.

12. Kim GT, Kim SH, Choi YS, Park YJ, Chung KR, Suk $\mathrm{KE}$, et al. Cone-beam computed tomography evaluation of orthodontic miniplate anchoring screws in the posterior maxilla. Am J Orthod Dentofacial Orthop 2009;136:628.e1-10; discussion 628-9.

13. Kuroda S, Yamada K, Deguchi T, Hashimoto T, Kyung HM, Takano-Yamamoto T. Root proximity is a major factor for screw failure in orthodontic anchorage. Am J Orthod Dentofacial Orthop 2007;131(4 Suppl):S68-73.

14. Kim SH, Kang SM, Choi YS, Kook YA, Chung KR, Huang JC. Cone-beam computed tomography evaluation of mini-implants after placement: is root proximity a major risk factor for failure? Am J Orthod Dentofacial Orthop 2010;138:264-76.

15. Kadioglu 0, Büyükyilmaz T, Zachrisson BU, Maino BG. Contact damage to root surfaces of premolars touching miniscrews during orthodontic treatment. Am J Orthod Dentofacial Orthop 2008;134:353-60.

16. Tronstad L. Root resorption--etiology, terminology and clinical manifestations. Endod Dent Traumatol 1988;4:241-52.

17. Lee KJ, Joo E, Kim KD, Lee JS, Park YC, Yu HS. Computed tomographic analysis of tooth-bearing alveolar bone for orthodontic miniscrew placement. Am J Orthod Dentofacial Orthop 2009;135:486-94.

18. Kim SY, Lim SH, Gang SN, Kim HJ. Crown and root lengths of incisors, canines, and premolars measured by cone-beam computed tomography in patients with malocclusions. Korean J Orthod 2013;43:271-8.

19. Kaya Y, Alkan Ö, Keskin S. An evaluation of the gingival biotype and the width of keratinized gingiva in the mandibular anterior region of individuals with different dental malocclusion groups and levels of crowding. Korean J Orthod 2017;47:176-85.

20. Kim MS, Lim SH, Jeong SR, Park JH. Maxillary molar intrusion and transverse decompensation to enable mandibular single-jaw surgery with rotational setback and transverse shift for a patient with mandibular prognathism and asymmetry. Am J Orthod Dentofacial Orthop 2020;157:818-31. 
21. Park JH, Choo H, Choi JY, Chung KR, Kim SH. Evaluation of strategic uprighting of the mandibular molars using an orthodontic miniplate and a nickel- titanium reverse curve arch wire: preliminary cephalometric study. Korean J Orthod 2021;51:179-88. 